This item was submitted to Loughborough's Research Repository by the author.

Items in Figshare are protected by copyright, with all rights reserved, unless otherwise indicated.

\title{
Shear viscosity measurements on Polyamide-12 polymers for laser sintering
}

PLEASE CITE THE PUBLISHED VERSION

http://dx.doi.org/10.1108/13552541311292709

\section{PUBLISHER}

(c) Emerald Group Publishing Limited

\section{VERSION}

AM (Accepted Manuscript)

\section{LICENCE}

CC BY-NC-ND 4.0

\section{REPOSITORY RECORD}

Haworth, Barry, Neil Hopkinson, David J. Hitt, and Xiaotao Zhong. 2015. "Shear Viscosity Measurements on Polyamide-12 Polymers for Laser Sintering”. figshare. https://hdl.handle.net/2134/17390. 
B. Haworth*, N. Hopkinson**, D.J. Hitt, X. Zhong,

Department of Materials, Loughborough University, UK.

\title{
Shear Viscosity Measurements on Polyamide-12 Polymers for Laser Sintering
}

\begin{abstract}
Purpose - Laser sintering kinetics and part reliability are critically dependent on the melt viscosity of materials, including polyamide 12 (PA-12). The purpose of this paper is to characterise the viscosity of $\mathrm{PA}-12$ powders using alternative scientific methods: constrained boundary flows (capillary rheometry) and rotational rheometry.

Design / methodology / approach - Various PA-12 powders were selected and characterised by both techniques. Measurement of molecular weight was also carried out to interpret the viscosity data.

Findings - Results demonstrate conventional pseudoplastic flow in all PA-12 materials. Zero-shear viscosity has been quantified by rotational rheometry; a notable observation is the striking difference between virgin / used PA-12. This is interpreted in terms of molecular weight and chain structure modifications, arising from polycondensation of PA-12 held at the bed temperature during laser sintering.

Research limitations / implications - Accurate zero-shear viscosity data provide scope for use in predictive computational models for laser sintering processes. Careful sample preparation and equipment operation are critical prerequisites for accurate rheological characterisation of PA-12 powders.

Practical implications - Differences in flow behaviour and molecular structure allow prediction and deeper understanding of process-property relationships in laser sintering, giving potential for further optimisation of material specification and in-process machine parameter control.

Originality / value - This is believed to be the first time that techniques other than MFR have been reported to measure the viscosity of PA-12 in a laser sintering context, noting the effects of pre-drying and molecular weight, then predicting differences between virgin / used powders in practical sintering behaviour.
\end{abstract}

Keywords - Laser sintering / nylon-12 / viscosity / rheology / polycondensation / molecular weight

Paper type - Research paper

* Mail address: Barry Haworth, Department of Materials, Loughborough University,

Loughborough, Leicestershire LE11 3TU, United Kingdom.

E-mail: B.Haworth@lboro.ac.uk

Tel: $\quad+441509223336$

Fax: $\quad+441509223949$

** Additive Manufacturing Research Group (AMRG), Wolfson School of Mechanical \& Manufacturing Engineering, Loughborough University, LE11 3TU, UK. 
Laser sintering (LS) processes for polymer-based materials are currently dominated by the use of polyamides, notably nylon / polyamide-12 (PA-12) powders supplied by the sintering equipment manufacturers. A critical parameter which determines sintering kinetics and the potential mechanical performance of sintered parts is the melt-state viscosity of the material. Despite the importance of this property in providing a link between material formulation, molecular weight and the subsequent mechanical behaviour in additive manufacturing, little has been published on the temperature and rate-dependent viscosity characteristics of PA-12 materials. Work in this area has almost exclusively been reported by researchers at the University of Louisville by employing melt flow rate (MFR) measurements (e.g. Davis et al, 2003, Hopkinson et al, 2005).

Combining an attractive range of mechanical properties with low material density, low water absorption and low shrinkage in shaping, PA-12 is an important material for LS in industrial applications and increasingly, in fundamental research (Childs and Tontowi, 2001; Tontowi and Childs, 2001). More recent studies have focused upon the effects of processing on structure development in PA-12 products, in order to optimise part durability and consistency. For example, investigations and analysis of crystal structure, microstructure and molecular weight of PA-12 to achieve high property reproducibility in PA-12 parts have been reported (Zarringhalam et al., 2006; Hopkinson et al, 2009). In addition, examples of materials-based research on PA-12 compounds in LS has been reported for PA-12 / HDPE polymer blends (Salmoria et al, 2008), aluminium particle reinforced PA-12 composites (Mazzoli et al, 2007) and PA-12 carbon black nanocomposites (Athreya et al, 2010).

Sintering processes for particulate materials can be predicted using a simple viscous model originally attributed to Frenkel (referenced by Muller et al, 2008), which relates process dynamics to particle viscosity and surface tension by:

$$
\frac{x}{r}=\left(\frac{3 \gamma}{2 \eta_{0} r}\right)^{1 / 2} t^{1 / 2}
$$

where $x$ is the joint length of particle coalescence relative to the original radius $(r), y$ is surface tension, $\eta_{0}$ is the zero-shear viscosity of the polymer, and $t$ is sintering time.

If particle radius and the viscosity parameter are held constant in this approach, the model is valid only for the early stages of sintering, and for viscous flows at low strain rates. Research has been reported to suggest that polymer particle sintering processes satisfy Newtonian flow criteria, consistent with the Frenkel model predictions (Rosenzweig and Narkis, 1981). Good agreement has also subsequently been obtained between practical measurement (using hot-stage microscopy) and Frenkel model predictions for PMMA and PP powders (Hornsby and Maxwell, 1992), although these authors used Troutonian (extensional viscosity) data for their predictions, as calculated from low strain rate shear viscosity data.

A development from the original Frenkel model was proposed by Eshelby (as noted by Bellehumeur et al, 1996) that recognises the importance of extensional flows in sintering processes. Subsequently, there has been a great deal of research aimed at generating more accurate and fundamental models of polymer sintering behaviour in other processes that involve particle coalescence, such as rotational moulding, powder coating and more recently, laser sintering. Some of these studies address the influence of polymer elasticity, the effects of different particle shapes and the progression of different phases within the sintering / coalescence processes; a notable chronology of these studies is cited for further 
reference (Bellehumeur et al, 1996). There is evidence that a high level of elasticity in polymers tends to slow the observed coalescence rates during sintering; an upper convected Maxwell (viscoelastic) model has been used to account for these observations (Vlachopoulos, 2008).

Modelling of the progression of sintering during laser sintering is particularly problematic because the process involves rapid changes of temperature and therefore variations in temperature dependent properties, including viscosity and surface free energy. Also, the process induces chain extension in polyamides, leading to an increase in molecular weight (Zarringhalam et al 2006) which in turn increases viscosity. As mentioned earlier, MFR has been used and developed as a useful tool to establish the state of feed material in the process (Davis et al 2003), however limitations of this method for modelling sintering must be recognised - in particular, MFR is conducted isothermally. Relationships between molecular weight and melt viscosity have also been reported (Shi et al, 2004), in a laser sintering context. Whilst the requirement for accurate and rigorous modelling of sintering processes is clear, most of the research activity has had a focus upon the viscosity of the polymer; it should be recognised that surface tension also has an important influence on sintering kinetics and the requirement to increase this parameter remains a challenge for polymer chemists.

Therefore, the objective of the research studies reported here is to provide a more comprehensive rheological characterisation of PA-12 powders, in order to understand the changes in behaviour that are likely to contribute to variability in processing and subsequent part performance. Techniques selected are based upon those which are commonly used in polymer engineering applications for other processes such as injection or rotational moulding. Due to the sensitivity of polyamides to the presence of moisture and differences in thermo-mechanical history, a further objective is to study these effects in practical rheometry, then to interpret the findings in terms of differences in molecular structure.

Ultimately, the mechanical properties of laser sintered components can only be optimised if these inter-relationships are carefully characterised, such that material specification and process parameters in laser sintering can be more carefully matched. A specific area for consideration in this respect is the use of 'recycled' powders (with different physical properties) from previous builds in laser sintering. Finally, a further benefit arising from accurate rheological data is the potential to utilise the properties data in predictive mode for computational analysis, as process sintering models are refined, developed further and potentially used to operate and control processes in real time.

\section{$2 \quad$ Materials \& Experimental Procedures}

\subsection{Materials}

In this project, different types of PA-12 materials were selected, as summarised in Table I (see also Figure 1). A key objective was to study differences in flow behaviour, so that a sample of 'used' powder (powder having been through a process cycle, therefore subjected to a laser sintering bed temperature of around $170^{\circ} \mathrm{C}$ for a period of up to $3-4$ hours) was included in the study. As a comparator, a commercial grade of PA-12 aimed at the injection moulding sector was also included and since polyamides are well known to be hygroscopic, an example of a powder without pre-drying was also included in the rheological analyses.

\subsection{Sample preparation and capillary rheometry}

The overall procedure for the experimental work is shown in Figure 2. Before the tests, the materials were dried to remove residual moisture; powders were placed on a piece of foil 
and dried in an air-oven for 1.5 hours at $80^{\circ} \mathrm{C}$. Following the pre-drying, samples were immediately sealed in air tight containers.

Studies of constrained shear flows over a range of shear rates were carried out using a Rosand $\mathrm{RH}-7$ twin-bore capillary rheometer at $200^{\circ} \mathrm{C}$; piston speeds were selected to achieve a shear rate range of $20-1000 \mathrm{~s}^{-1}$. Simultaneous experiments were conducted using dies with length / diameter dimensions of $16 / 1$ and $0 / 1$ (dimensions in $\mathrm{mm}$ ). The first of these is used to obtain shear flow data, whilst the orifice ('zero length') die is used to achieve corrected values of shear viscosity, by eliminating the energy contributions associated with the extensional 'converging' flows at the die entrance. Raw data from the orifice die can also be used to obtain extensional flow data, according to the method published elsewhere (Cogswell, 1994). For all experiments, data were exported via the 'Flowmaster' software system and subsequently exported to customised databases for results display purposes. Two complete runs per sample were conducted; the barrel and dies were cleaned thoroughly following each experiment.

\subsection{Compression moulding and rotational rheometry}

Rotational rheometry involves subjecting a sample of molten material to a continuous, steady-state shear deformation, in this case between two parallel plates, one of which rotates with respect to the other. Rather than initiate testing on powder or granulated samples, disc-shaped specimens (dimensions: $25 \mathrm{~mm}$ diameter and $1 \mathrm{~mm}$ thickness) were prepared by compression moulding. A disk-shape mould was filled with the PA-12 powders, clamped by two steel plates and inserted into a compression moulding press; machine parameters (temperature $200^{\circ} \mathrm{C}$ and pressure 10 tonnes) were maintained for 3 minutes, followed by an additional 3 minutes cooling time. For the flow analysis by rotational rheometry, a parallel plate system was used (Advanced Rheometric Expansion System (ARES), TA Instruments, USA) using samples in steady shear deformation (continuous rotation in one direction). A convection oven was used for heating to the test temperature $\left(20{ }^{\circ} \mathrm{C}\right)$; samples were held under a nitrogen blanket to avoid oxidative degradation.

\subsection{Material characterisation}

In order to estimate surface energy data, contact angle measurement was carried out on the compression moulded samples of PA-12 (virgin and used) at Loughborough Materials Characterisation Centre (LMCC). An OCA-series contact angle system (Dataphysics Instruments $\mathrm{Gmbh}$ ), was employed and two solvents, water and diiodomethane, were used in the tests carried out at ambient conditions. Note that injection moulded and un-dried material samples were not subject to contact angle measurement as these represented material conditions that were un-realistic in the laser sintering process and therefore data from these would not be valid for inclusion in sintering models.

Molecular weight and polydispersity (molecular weight distribution) data were investigated using gel permeation chromatography (GPC) techniques carried out at Smithers Rapra (Holding \& Forsyth, 2009). Polyamide samples were analysed in solution and chromatography was carried out at an elevated temperature; data were collected and analysed using Polymer Laboratories 'Cirrus' software to produce molecular weight distribution curves. 
Results \& Discussion

\subsection{Shear flow behaviour at high deformation rates - capillary rheometry}

Results from the constrained shear flow analysis are presented first (see Figures 3-5). Data have been analysed according to the simple power law model for shear flow (Birley et al, 1991; Cogswell 1994), which can be converted into logarithmic form:

$$
\log \tau=\log k+n \cdot \log \gamma
$$

$\tau$ is shear stress, $\gamma$ is shear (strain) rate and $\mathrm{k}, \mathrm{n}$ are the constants describing flow in the power law region (consistency index and power law index, respectively).

Therefore, from a plot of shear stress vs. shear rate (Figure 3), estimated values of power law constants ( $k$ and $n$ ) can be obtained for PA-12. These are shown in Table II. The power law index for the used PA-12 material is estimated at 0.52 , indicating conventional pseudoplastic behaviour, as observed in Figure 3. Over the range of shear strain rates studied, a high level of linearity was observed for the used PA-12, giving confidence in the ability to predict shear viscosity as a function of shear rate. This type of response is considered typical for polymers of high molecular weight, for which shear flow behaviour is dependent upon the influence of transient-state physical entanglements within the molecular network, which influence the measured viscosity under given conditions.

The data for the other materials have also been analysed according to the power law assumption, on the same plot. The degree of correlation is significantly lower and moreover, the predicted values of $n$ (exceeding unity) are unrealistic. This is due to the anomalous flow behaviour in the low shear rate range, where an apparent reduction in shear viscosity is observed (Figures 4, 5). This observation is thought to represent an inaccurate representation of the flow behaviour of the PA-12 polymers and is much more likely to be attributed to an experimental flaw associated with pre-heating, air entrapment, or a progressive change to the polyamide molecular structure during the course of the rheological measurements.

The most striking observation in Figures 3-5 is the difference in flow behaviour between the used PA-12 and the other materials, best illustrated by plots of shear viscosity versus shear rate (Figures 4,5 ). Valid measurements are probably restricted to the shear rate range $10^{2}-10^{3} \mathrm{~s}^{-1}$ in Figure 4 for the reasons given above, since the shear viscosity of high polymers is known to tend towards a limiting value at low stress: the so-called zeroshear viscosity $\left(\eta_{0}\right)$. The virgin LS powder has a marginally higher viscosity than the injection moulding grade at $200{ }^{\circ} \mathrm{C}$, across the shear rate range of interest; the data are qualitatively similar however, implying that the molecular structures of these materials are related. Once fully melted, the LS grade would be expected to show similar flow characteristics in scaledup processes.

The influence and importance of pre-drying is also apparent from the data: shear viscosity of PA-12 (at a shear rate of $100 \mathrm{~s}^{-1}$ ) is around 270-320 Pa.s, compared to 130-160 Pa.s for the powder that was not pre-dried. Although the trends appear similar in Figures 45 , it should be noted that a logarithmic scale for viscosity is always used on the ordinate, so that the absolute values of viscosity (which might be used for computational analysis) are significantly different. Plotting viscosity versus shear stress (Figure 5) exaggerates the distinction in flow behaviour between virgin and used powders. Water molecules are attracted to polyamides due to their hydrophilic nature and the dominant effect of hydrogen bonding between the molecular chains; the presence of water tends to decrease inter- 
molecular attraction, such that physical properties (modulus and strength in the solid-state; viscosity in the melt-state) are modified by moisture content. In addition however, chemical reaction is also possible in polycondensation-type materials such as polyamides, whereby reductions in molecular weight can occur due to hydrolysis. In this case, it is suggested that the observed reduction in viscosity for the un-dried PA-12 is predominantly due to physical absorption of water molecules, rather than by chemical hydrolysis.

\subsection{Shear flow behaviour at low deformation rates - rotational rheometry}

The ARES parallel plate rheometer was used to obtain viscosity data over a lower range of shear strain rates; rotation speed (for given sample dimensions) determines shear rate, the measured torque is directly proportional to shear stress, so that polymer viscosity data can be computed from these variables (e.g. Macosko, 1994). Figure 6 shows viscosity data for the three PA-12 materials. In all cases, limiting values of zero shear viscosity $\left(\eta_{0}\right)$ are evident as shear rate tends to zero; numerical values have been estimated using the Ellis model and are presented in Table III. As would be anticipated from capillary rheometry, the virgin LS PA-12 material and the injection moulding grade yield similar values of $\eta_{0}$, whereas the used powder shows $\eta_{0}$ values of over a magnitude higher (exceeding $5000 \mathrm{~Pa}$.s). Whilst the reasons for this difference in viscosity will be discussed later (Section 3.4), it is useful at this stage to combine the data from each technique to form a composite plot of viscosity - shear rate data covering three orders of magnitude (Figure 7). In principle, viscosity data should be independent of equipment, specimen geometry and measurement principle, so that a continuous plot would be anticipated. Agreement here is reasonable, although it is clear that there are some experimental factors associated with either technique, which invalidate data points within the high end of the strain rate (for rotational rheometry, where flow instability occurs) and also the low end of the range investigated by capillary rheometry (see earlier comments). In addition, there may be discrepancy between each data set (rotational and capillary flows ) due to the additional thermal history associated with the compression moulding phase to produce the specimens required for the latter technique.

The overall response of the polyamides shows near-Newtonian behaviour at low shear rates, with viscosity tending to a limiting value $\left(n_{0}\right)$ as shear rate tends towards zero (Figures 6,7). The Newtonian plateau is less clearly defined for the used PA-12, due to its higher molar mass (see Section 3.4). Across the higher shear rate range in capillary rheometry, pseudoplastic flow is observed and where the bi-logarithmic plot becomes linear, the flow response can be approximated by simple power law behaviour. Over the entire shear rate range, an appropriate flow model must be able to account for each type of response, over a continuous shear rate range, for appropriate computational analysis to be carried out. Flow data from the low shear region are used to predict sintering behaviour using a simple viscous model (Frenkel) in Section 3.5.

\subsection{Extensional flow behaviour inferred from capillary rheometry data}

When using capillary rheometry, the melt convergence at the die entrance can be approximated to a constrained extensional flow, so that information on the tensile melt flow properties can be inferred by data collected from an orifice ('zero length') die (Cogswell, 1972; 1994). Extensional flow data for PA-12 materials (plotted as extensional viscosity versus tensile strain rate) are shown in Figure 8 and these data are combined with the shear flow data in Figure 9. Extensional viscosity decreases with an increase of extensional stress for all PA-12 samples; 'tension thinning' behaviour is typical of linear polymers, especially those with relatively low molar mass. However, since the stress (hence viscosity) data are computed from measurements of pressure from an orifice (zero-length) die, which are always significantly lower than equivalent pressure data from long dies, a higher degree of variation is usually apparent in the data points. Moreover, any unstable flow or pressure fluctuations in capillary rheometry will affect the pressure readings above the orifice die to a 
greater extent. Since extensional viscosity is a strong function of stress, it is not a straight forward task to predict a limiting value at the low strain rates observed in laser sintering; this observation has relevance to any future attempts to use extensional flow data in predictive mode.

Overall, the main trend evident from Figure 8 is the higher extensional viscosity of the used PA-12 material, thus reinforcing the earlier comments made regarding the influence of molecular weight on the measured viscosity characteristics.

\subsection{Molecular weight dependence of shear viscosity}

Based on the results from GPC analysis and rheometry, the molecular weight dependence of shear viscosity can be investigated for PA-12. Molecular weight data are shown in Table IV. For most commercial plastics above the critical molar mass, the value of zero shear viscosity depends on weight average molecular weight (Lobo and Bonilla, 2003), given by a power law of the form:

$$
\eta_{0}=C \cdot M w^{a}
$$

Taking the logarithmic form:

$$
\log \left(\eta_{0}\right)=\log C+a \cdot \log (M w)
$$

Our data can be interpolated to estimate the power law constants $(\mathrm{C}$ and $\mathrm{a}$ ) including index (a), which can then be used for predictive purposes if the molecular weight of PA-12 samples is known. Plots are shown in Figure 10 illustrating the data according to a linear fit, based on the relationships given above. Theoretical values of index (a) for high polymers are usually quoted in the range $3.4-3.6$; the exponent for PA-12 at zero shear rate $(n=2.33)$ is lower than anticipated, since a limited number of samples were analysed; clearly, more data points would be required for a more accurate representation.

The main point however is the clear increase in molecular weight $(\mathrm{Mw})$ and polydispersity (Mw/Mn) for the 'used' material which has passed through a laser sintering cycle. This is thought to be attributed to a solid-state polycondensation reaction taking place within the inert atmosphere of the sintering equipment, resulting in chain extension in PA-12. An increase in Mw increases the mechanical properties of artefacts (Hitt et al, 2011), yet compromises the ability of PA-12 to be sintered effectively, by increasing polymer melt viscosity.

\subsection{Surface energy data and prediction of sintering behaviour}

Having obtained accurate viscosity data, it was of interest to obtain measurements of surface energy in PA-12 materials, including differences between virgin and used materials, in order to investigate the use of the physical properties data in predictive models for sintering behaviour. These were performed by contact angle measurements and results are given in Table V. Surface energy data are typical of what might be expected from polyamides; the differences observed between samples of virgin / used powders can be attributed to the contributions from both the dispersive and polar components. The reasons for these differences remain speculative, but are probably influenced by the presence of additives in the surface regions, the difference in additive concentration between virgin / used powders and the additional heat history of the 'used' PA-12 materials. Table V also quotes other values of surface energy (or surface tension, in the case of molten polyamides) taken from literature citations. It is clear that our measured $y$-value for virgin PA-12 appears high, in comparison to other reported data. When using sintering models however, it is more appropriate to use property values appropriate to the actual process temperature range. In our laser sintering work, the precise temperatures to which the powders are subjected are unknown; thermal gradients will be evident within the powder particles and steady-state 
conditions will not be achieved. Therefore, the use of single point values in sintering models is strictly invalid, so that predictions will always be approximations, yet these do retain some value for comparative purposes.

From the information in Table $\mathrm{V}$, we might speculate that surface tension values in the range $25-30 \mathrm{mN} \mathrm{m}^{-1}$ might be appropriate for the virgin PA-12 materials, in typical laser sintering processes at high temperature. Taking the $\eta_{0}$ values for virgin / used powders from Table III (390 / $5095 \mathrm{Ns} \mathrm{m}^{-2}$, respectively) and a particle size of $56 \mu \mathrm{m}$ (equivalent diameter), the Frenkel model can be used to predict 'sintering times' using these values, to produce the results shown in Figure 11. $n_{0}$ values for intermediate virgin / used PA-12 mixes have been predicted from the measured viscosity data in Table III, using the power law constants (C and a) derived using equations 3 and 4 . Despite its lack of fundamental rigour and its basis on Newtonian flow criteria, previous work has shown that the Frenkel model can be used successfully as a predictive tool for the sintering of semi-crystalline particles (e.g. Hornsby and Maxwell, 1992). Whilst accepting that the use of a relatively simple viscous model is unlikely to lead to wholly accurate predictions, comparative data from sensitivity analysis predictions of this type are of significant value.

Figure 11 demonstrates that the change in shear viscosity (between virgin / used PA12) dominates the predicted sintering behaviour. Quantitative values on the ordinate $(x / r$, dimensionless neck radius) have significance up to values of around 1.4: the times required to achieve different levels of sintering can then be predicted from the $\mathrm{x}$-axis. These data demonstrate the importance of zero-shear viscosity of polymers used in sintering; this parameter is temperature-dependent but since particle temperature is transient, position dependent and not easily controlled in laser sintering processes, the most important parameter that influences $\eta_{0}$ is the molecular weight of the PA-12 material (Figure 11). This observation therefore has practical relevance to the choice of polymers used in laser sintering and the effect of molecular weight on viscosity and sintering kinetics. Clearly, a relatively low molecular weight favours sintering, but once parts are formed using LS, higher molecular weight promotes enhancement of physical properties.

Overall, viscosity measurement and process simulation is an area of ongoing research that acts as a challenge for rheologists interested in modelling flow behaviour in polymer processes. For example, predictive modelling of shear viscosity for plastics injection moulding simulation is well developed: there are several viscous models available to account for such behaviour, including the Ellis model (which accounts for the constant viscosity region at low shear rates), the Carreau model (which also accounts for the tendency to observe a limiting viscosity at very high rates of shear) and the Cross-WLF model, based upon up to seven model coefficients, including the power law index (n) from the linear region. Whilst similar rheological predictions in polymer sintering are also developing (Bellehumeur et al, 1996; Muller et al, 2008), these are outside the immediate scope of the research presented here. Nevertheless, the results presented in this paper provide an indication of the important physical property requirements for predictive modelling of sintering processes using the viscous Frenkel model; the importance of PA-12 thermal history and viscosity dependence upon molecular weight, have been demonstrated.

\section{Conclusions}

This paper examines a number of rheological measurements on PA-12 powders using constrained boundary flows (capillary rheometry) and low shear measurements in rotational shear. Results demonstrate that conventional pseudoplastic flow behaviour occurs in PA-12, with melt viscosity decreasing when shear rate is increased; the overall response of molten PA-12 powders is quantitatively similar to as-supplied (virgin) materials intended for injection moulding. The influence of pre-drying is also significant; a measured reduction in shear 
viscosity is apparent if the powders are not pre-dried. Shear viscosity values between 100400 Pa.s were typically observed across a shear rate range of $10^{2}-10^{3} \mathrm{~s}^{-1}$, for all except the used PA-12 powder. Some anomalous effects were apparent in the capillary rheometry experiments, where an apparent decrease in shear viscosity was observed at the lower end of the shear rate range, which is likely to be associated with pre-drying, thermal history and competing effects on the molecular structure of polyamides during the flow analysis.

Results from rotational rheometry have allowed values of zero-shear viscosity to be quantified, giving scope to use these in predictive models for sintering processes. A notable observation throughout this research is the significantly different flow behaviour between virgin and used PA-12; the latter material has a higher shear viscosity and retains a strong pseudoplastic response even at the lowest shear strain rates investigated. This behaviour has been interpreted by changes in molecular weight and chain structure arising from polycondensation effects, which occur whilst PA-12 is held at the bed temperature during laser sintering. Differences in viscosity will lead to variability in sintering behaviour and properties of laser sintered parts. Viscosity data have therefore been interpreted in terms of molecular weight data for the powders. Whilst a direct increase in $\mathrm{Mw}$ is likely to enhance mechanical properties of laser sintered parts, the use of 'recycled' powder of increased molecular weight will change viscosity considerably, with possible implications on sintering kinetics when virgin / used powders are mixed, as demonstrated by data predictions using the Frenkel viscous model to simulate sintering behaviour.

\section{Acknowledgements}

We would like to acknowledge the research funding from the Engineering and Physical Sciences Research Council (EPSRC) of Great Britain, via the Innovative Manufacturing and Construction Research Centre (IMCRC) at Loughborough University. Other contributions, notably those from our research partners / industrial sponsors (EOS Gmbh, Burton Snowboards), Anurag Pandey at Loughborough University (rotational rheometry) and Smithers-Rapra (GPC analysis) are also acknowledged with thanks.

\section{References}

Athreya, S.R., Kalaitzidou, K. and Das, S. (2010), "Processing and characterization of a carbon-black filled electrically conductive nylon-12 nanocomposite produced by selective laser sintering", Mat. Sci. \& Eng. A: Vol. 527, pp. 2637-2642.

Bellehumeur, C.T., Bisaria, M.K. and Vlachopoulos, J. (1996), "An Experimental Study and Model Assessment of Polymer Sintering”, Polym. Eng. Sci., Vol. 36, pp. 2198-2207.

Birley A.W., Haworth B. and Batchelor J. (1991), "Physics of Plastics", Carl Hanser, Munich.

Brandrup, J., Immergut, E.H. and Grulke, E.A. (1999), "Polymer Handbook, $4^{\text {th }}$ Ed.", Wiley, New York.

Childs, T.H.C. and Tontowi, A.E. (2001), Selective laser sintering of a crystalline and a glass-filled crystalline polymer: experiments and simulations, Proc. I. Mech. E., Vol. 215, pp.1481-1495.

Cogswell, F.N. (1972), "Converging Flow of Polymer Melts in Extrusion Dies", Polym. Eng. Sci., Vol. 12, pp. 64-73.

Cogswell, F.N. (1994), "Polymer Melt Rheology - A Guide for Industrial Practice", Woodhead Publishing, Cambridge, UK. 
Davis, K.R., Gornet, T.J. and Richardson, K.M. (2003), "Material testing method for process control of direct manufacturing in the SLS process", Proceeding of the $1^{\text {st }}$ International Conference on Advanced Research in Virtual and Rapid Prototyping, Leiria, Portugal, October 2003.

Hitt, D.J., Haworth, B. and Hopkinson, N. (2011), Fracture Mechanics Approach to Compare Laser Sintered Parts and Injection Mouldings of Nylon-12, Proc. I. Mech. E. Part B, Journal of Engineering Manufacture, In-Press.

Holding, S and Forsyth, T. (2009), Smithers-Rapra Confidential Technical Report to Loughborough University.

Hopkinson, N., Hague, R.M.J. and Dickens, P.M.(2005), Rapid Manufacturing an Industrial Revolution for the Digital Age, John Wiley and Sons Ltd., New York, ISBN 13 978-0-47001613-8

Hopkinson, N, Majewski, C.E. and Zarringhalam, H. (2009), Quantifying the degree of particle melt in Selective Laser Sintering ${ }^{\circledR}$, CIRP Annals - Manufacturing Technology, Elsevier, doi: 10.1016/j.cirp.2009.03.001, pp 197-200.

Hornsby, P.R. and Maxwell, A.S. (1992), "Mechanism of sintering between polypropylene beads", J. Mat. Sci., Vol. 27, pp. 2525-2533.

Lobo, H. and Bonilla, J.V. (2003), "Handbook of Plastics Analysis", Marcel Dekker Inc., New York.

Macosko, C.W. (1994), "Rheology Principles, Measurements and Applications", VCH Inc., New York.

Mazzoli, A., Moriconi, G. and Pauri, M.G. (2007), "Characterisation of an aluminium-filled polyamide powder for applications in selective laser sintering", Materials and Design Vol. 28, pp. 993-1000.

Muller, J-D, Bousmina, M. and Maazooz, A., (2008), "2D-Sintering Kinetics of Two Model Fluids as Drops", Macromolecules, Vol. 41, pp.2096-2103.

Rosenzweig, N. and Narkis, M. (1981), "Observation and analysis technique for studying sintering of polymeric particles", J. Appl. Polym. Sci., Vol. 26, pp. 2787-2789.

Salmoria, G.V., Leite, J.L., Paggi, R.A., Lago, A. and Pires, A.T.N. (2008), "Selective laser sintering of PA12 / HDPE blends: effect of components on elastic / plastic behaviour", Polymer Testing, Vol. 27, pp. 654-659.

Shi, Y., Li, Z., Sun, H., Huang, S. and Zeng, F. (2004), "Effect of the properties of the polymer materials on the quality of selective laser sintering parts", Proc. Inst. Mech. Eng. Part L: J. Materials: Design and Applications, Vol. 218, pp. 247-252.

Tontowi, A.E. and Childs, T.H.C. (2001), "Density prediction of crystalline polymer sintered parts at various powder bed temperatures", Rapid Prototyping Journal, Vol. 7, pp. 180-184.

Vlachopoulos, J., Takacs, E. (2008), "The role of viscosity, elasticity and surface tension on polymer particle coalescence", Presented at PPS USA Regional Conf., Charleston SC, USA. 
Zarringhalam, H., Hopkinson, N., Kamperman, N.F. and de Vlieger, J.J., (2006), "Effects of processing on microstructure and properties of SLS Nylon 12", Materials Science and Engineering: A, Vol. 435-436, pp.172-180. 


\section{Figures}

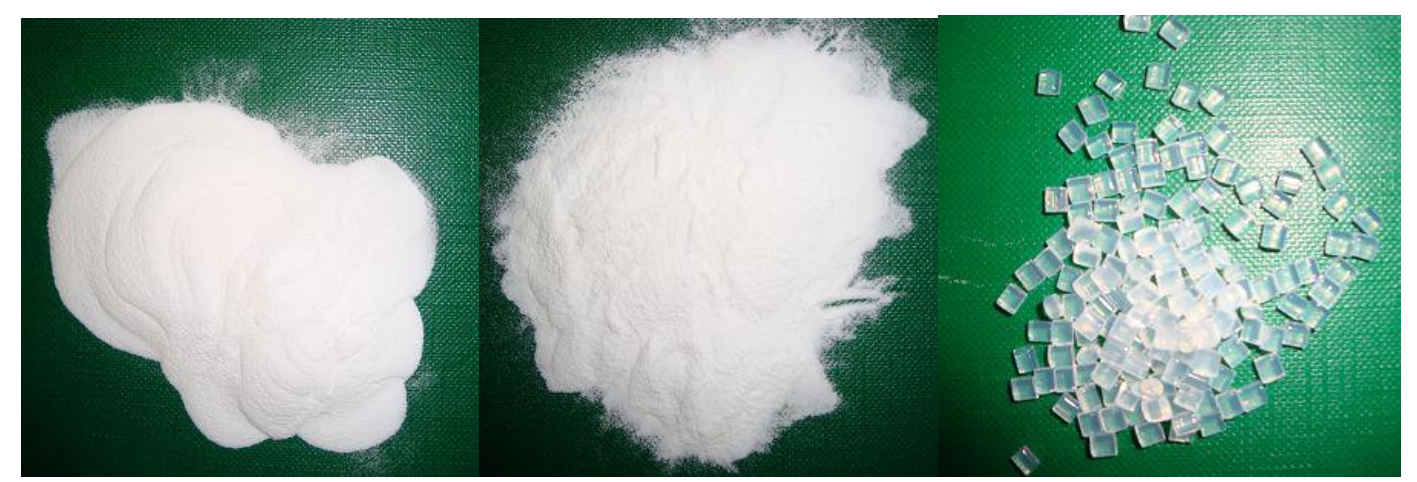

Figure $1 \quad$ PA 12 samples used in the study: (a) PA 12 used powder; (b) PA 12 virgin powder; (c) PA 12 granules for injection moulding

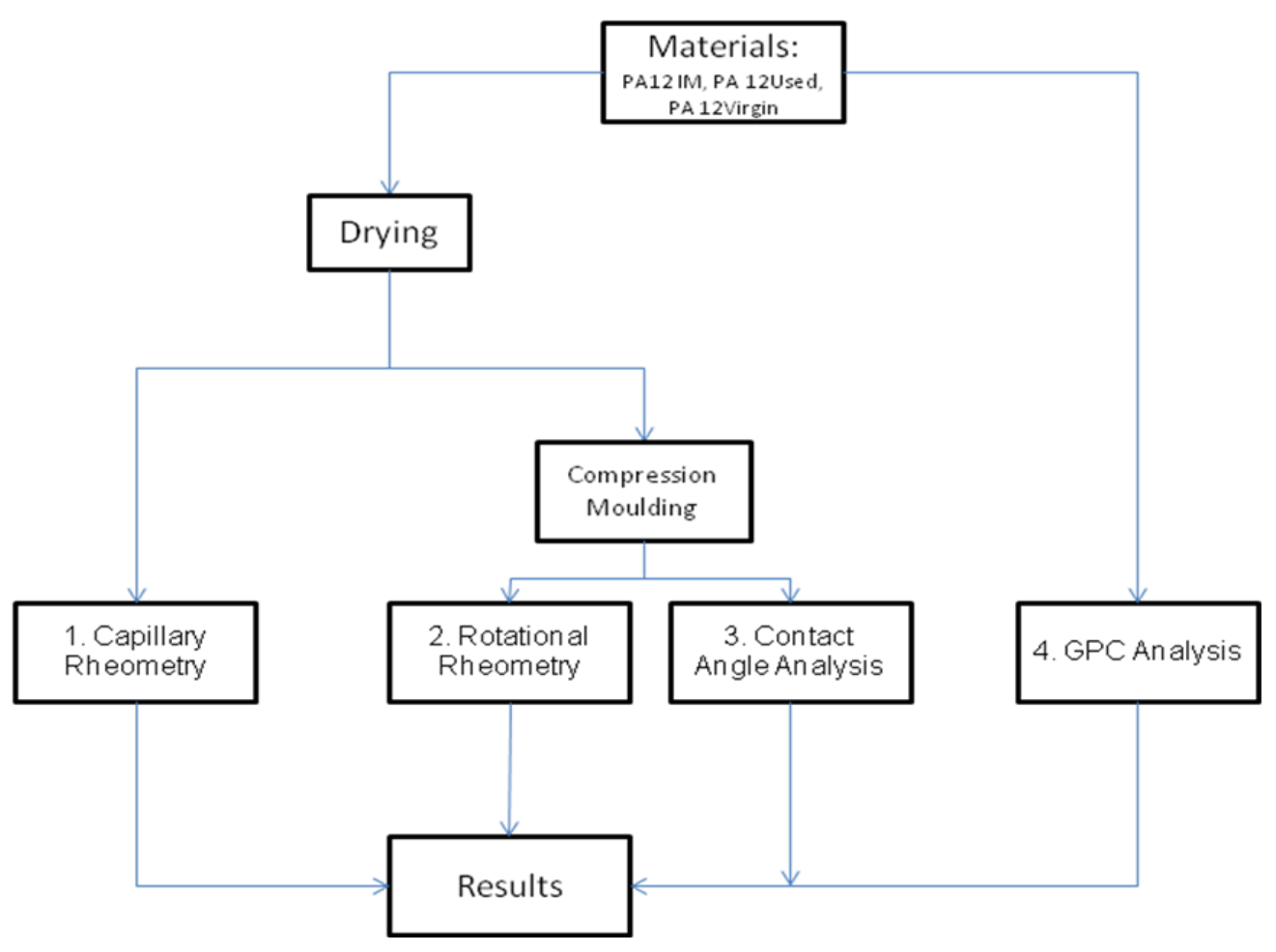

Figure 2 Flow chart: the procedures used in the experimental work. 


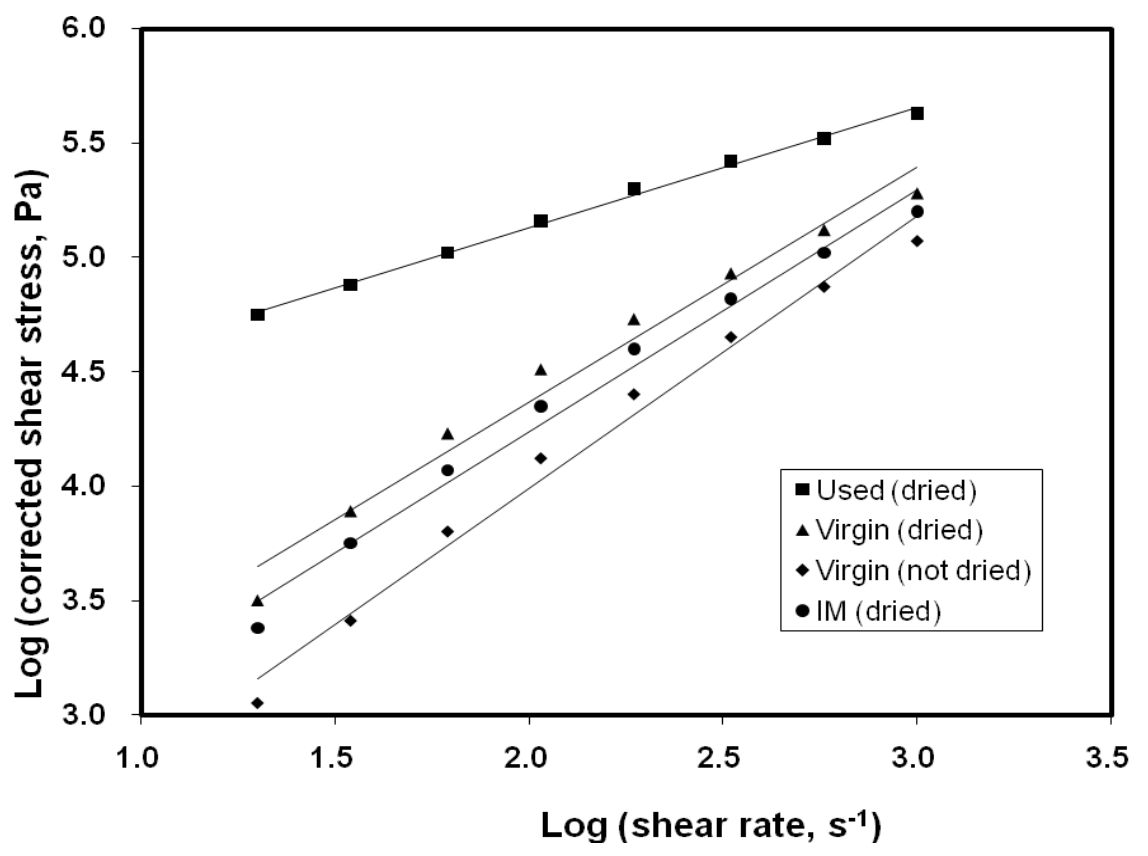

Figure 3 Shear viscosity data from capillary rheometry at $200{ }^{\circ} \mathrm{C}$ for PA-12 polymers, plotted as shear stress versus shear rate (power law fit). [Legend: in all figures, 'used' and 'virgin' refer to PA-12 powders and 'IM' to the injection moulding grade.]

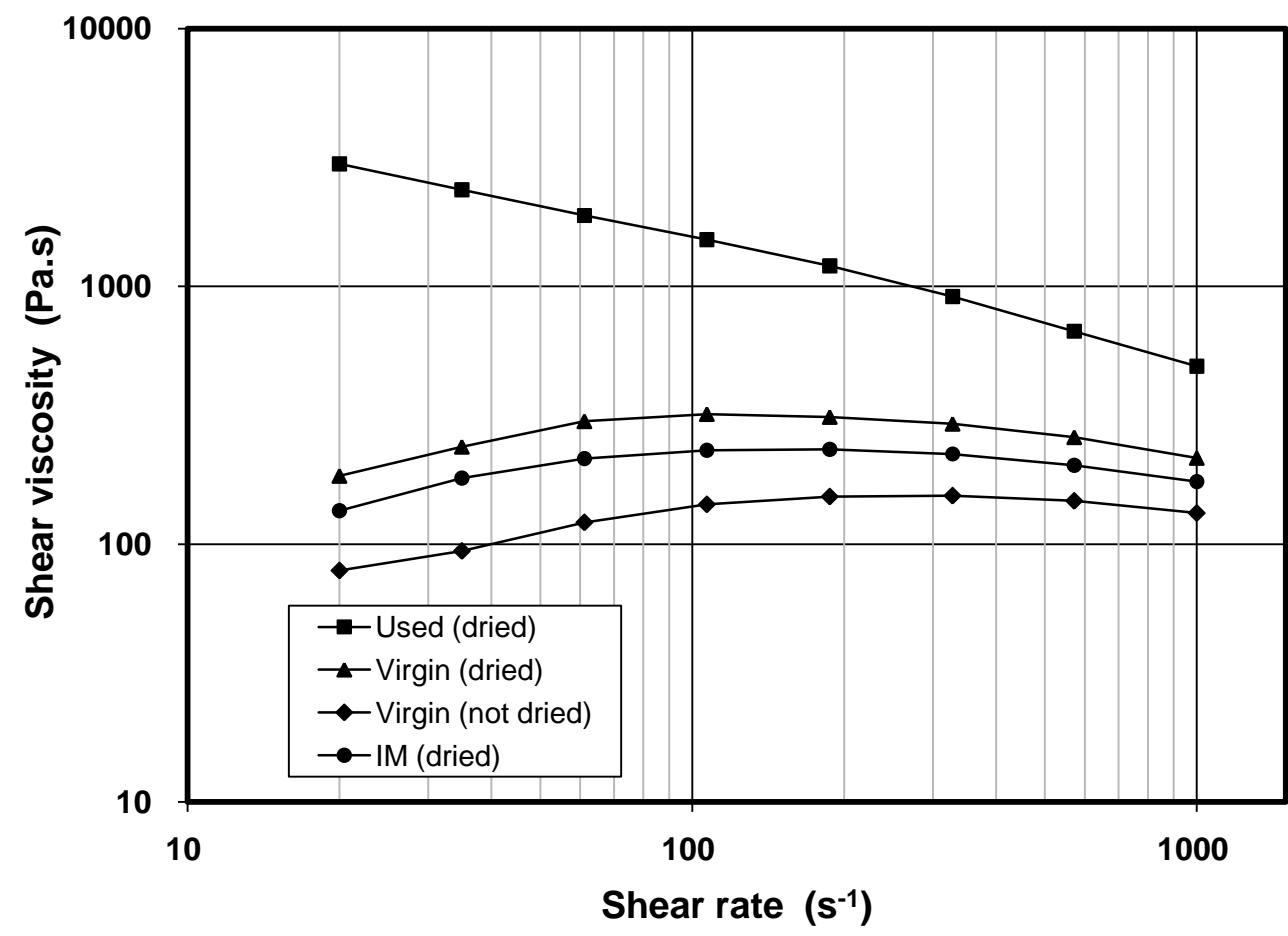

Figure 4 Shear viscosity data from capillary rheometry at $200{ }^{\circ} \mathrm{C}$ for $\mathrm{PA}-12$ polymers, plotted as shear viscosity versus shear rate. 


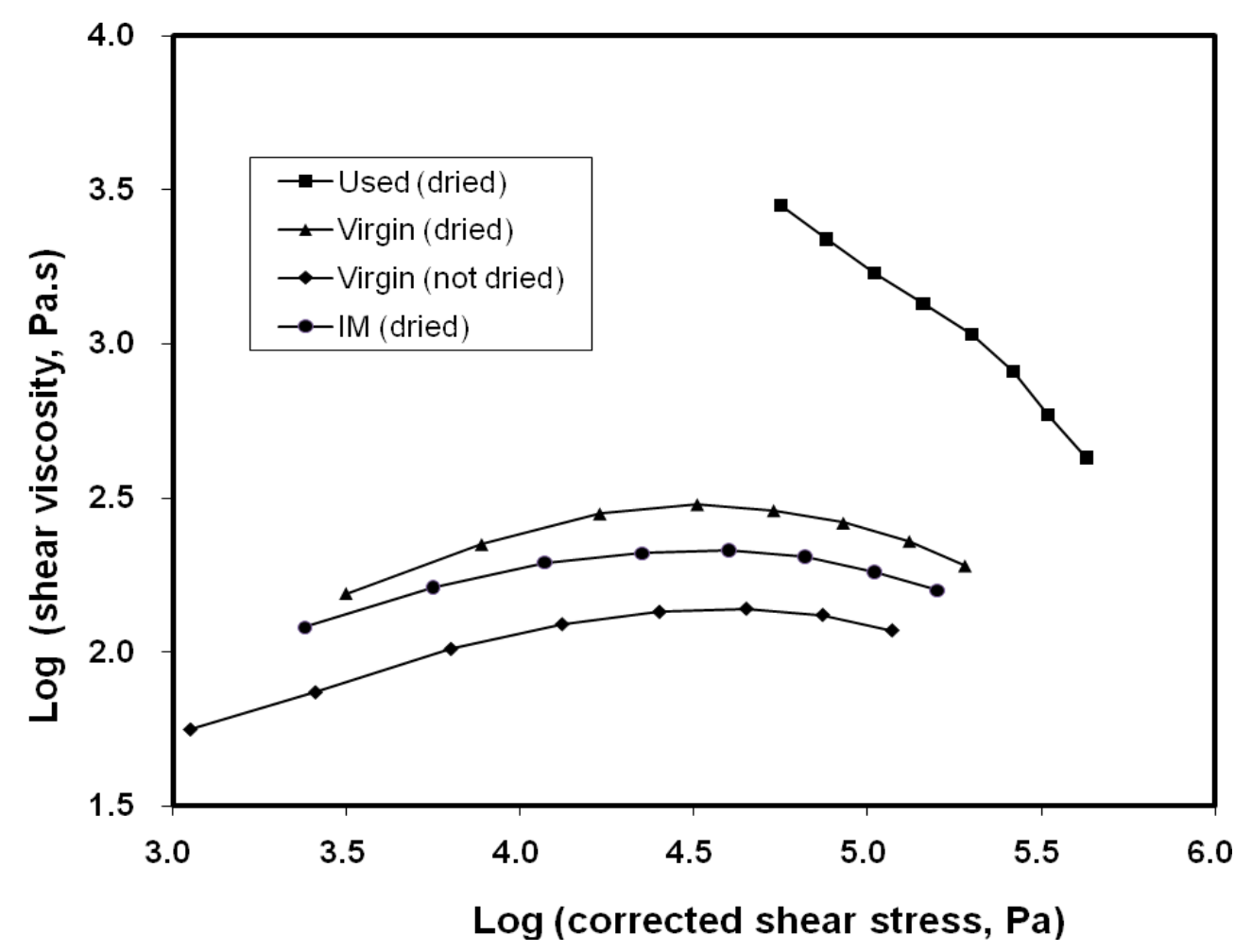

Figure 5 Shear viscosity data from capillary rheometry at $200{ }^{\circ} \mathrm{C}$ for $\mathrm{PA}-12$ polymers, plotted as shear viscosity versus shear stress.

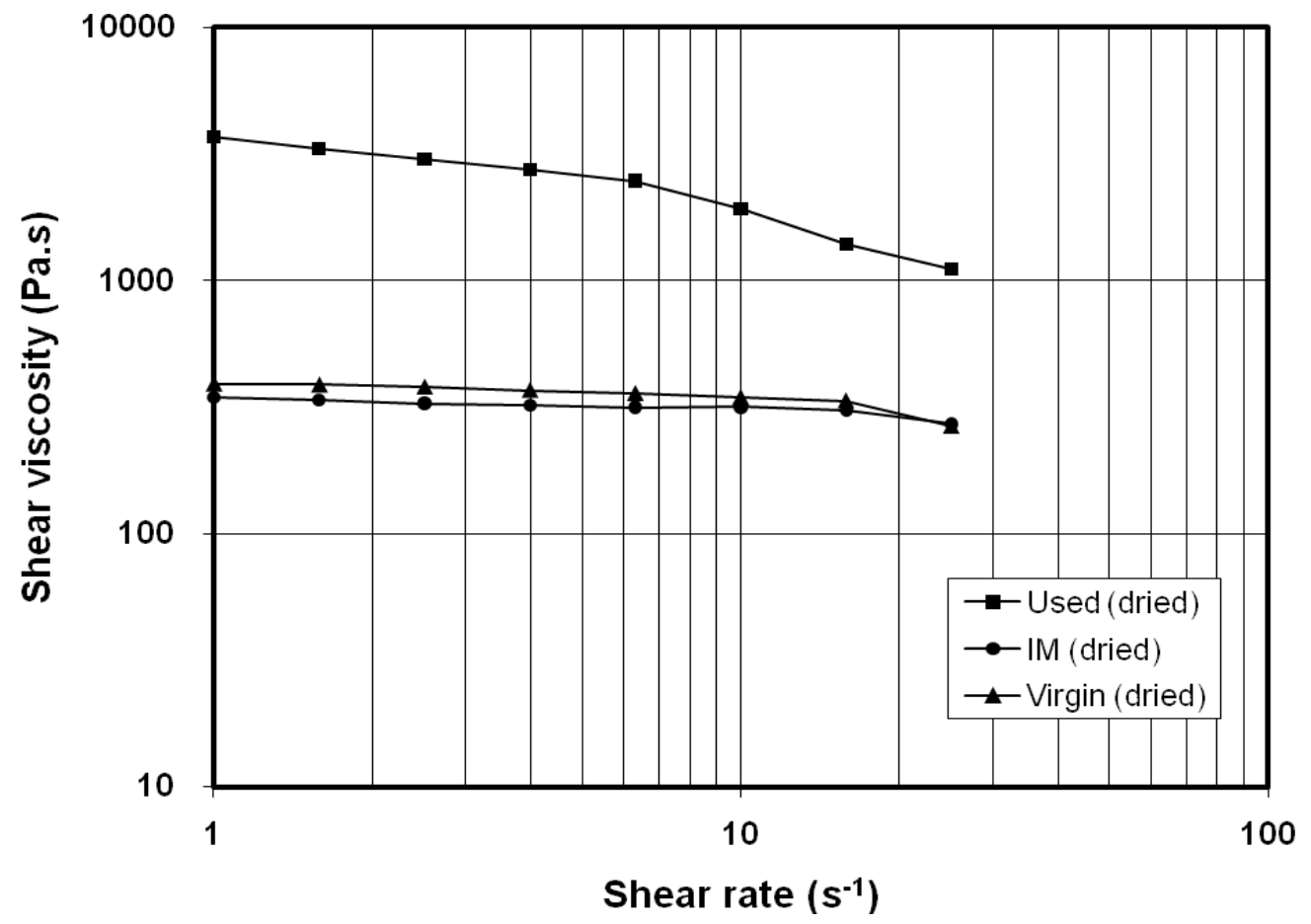

Figure 6 Shear viscosity data from rotational rheometry at $200{ }^{\circ} \mathrm{C}$ for PA-12 polymers (low shear rate range), plotted as shear viscosity versus shear stress. 


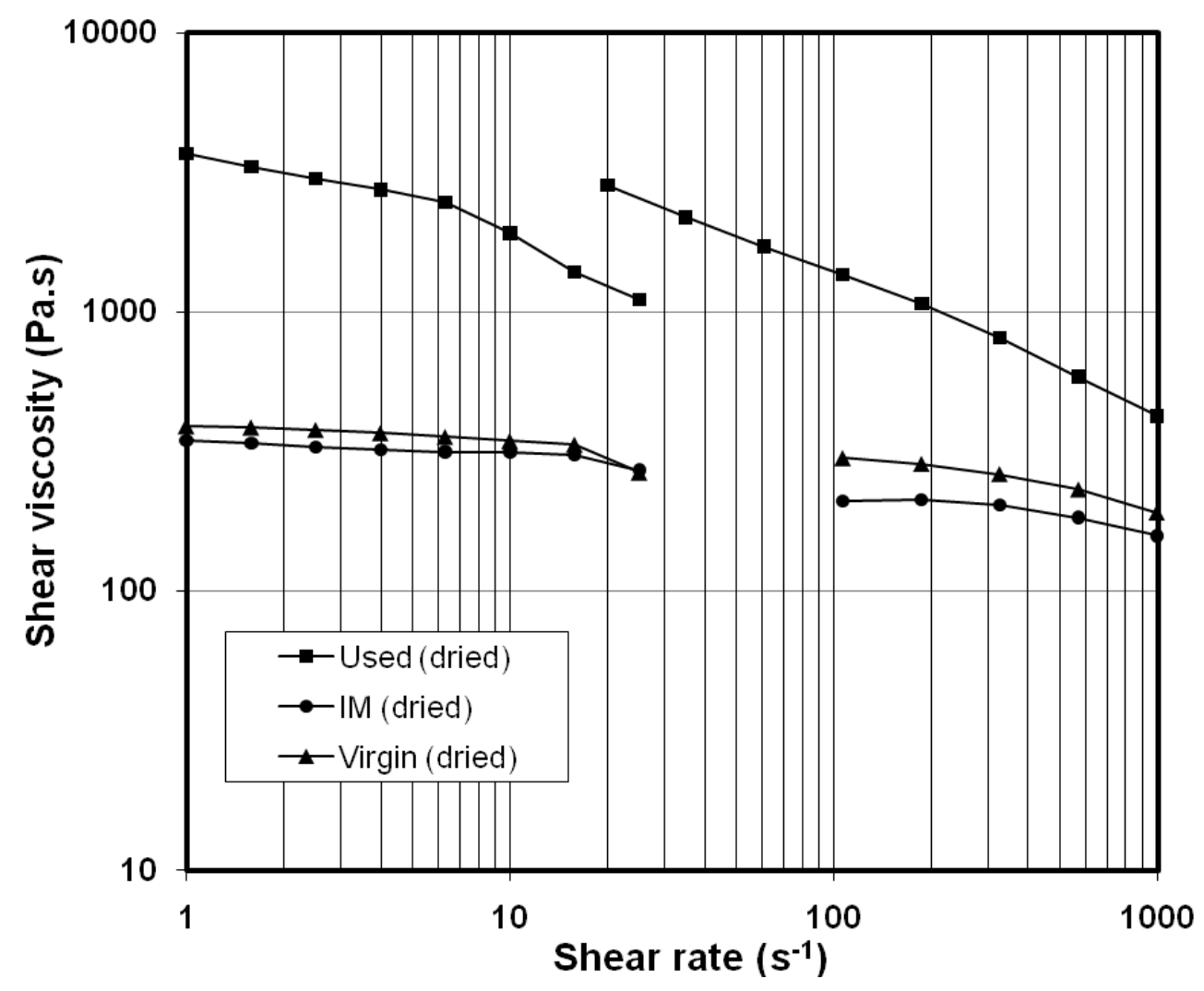

Figure $7 \quad$ Composite plot of shear viscosity data for PA-12 polymers: low shear rate range from rotational rheometer, high shear rate range from capillary rheometer.

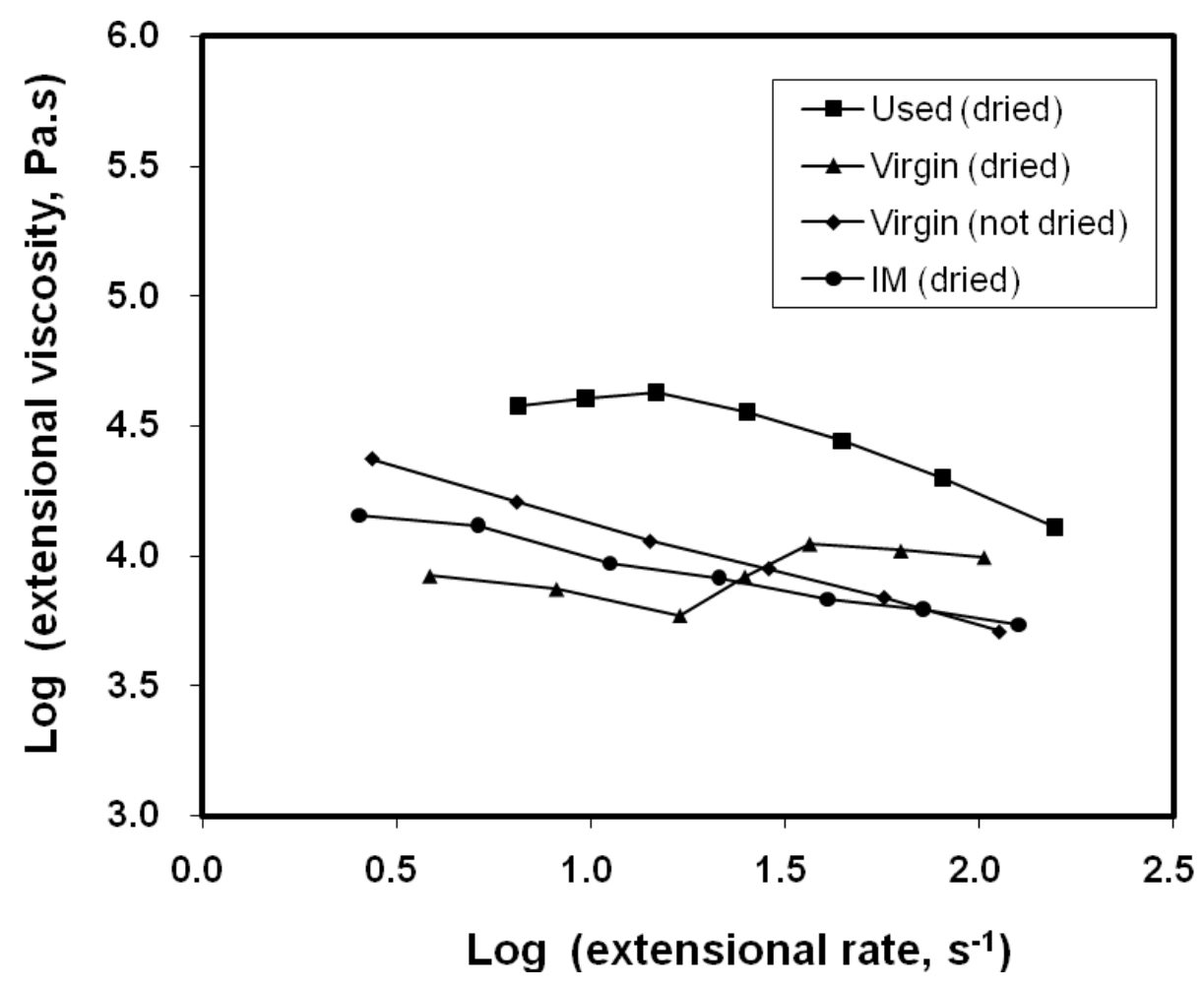

Figure $8 \quad$ Extensional viscosity data inferred from capillary rheometry at $200{ }^{\circ} \mathrm{C}$ for PA12 polymers, plotted as extensional viscosity versus tensile strain rate. 


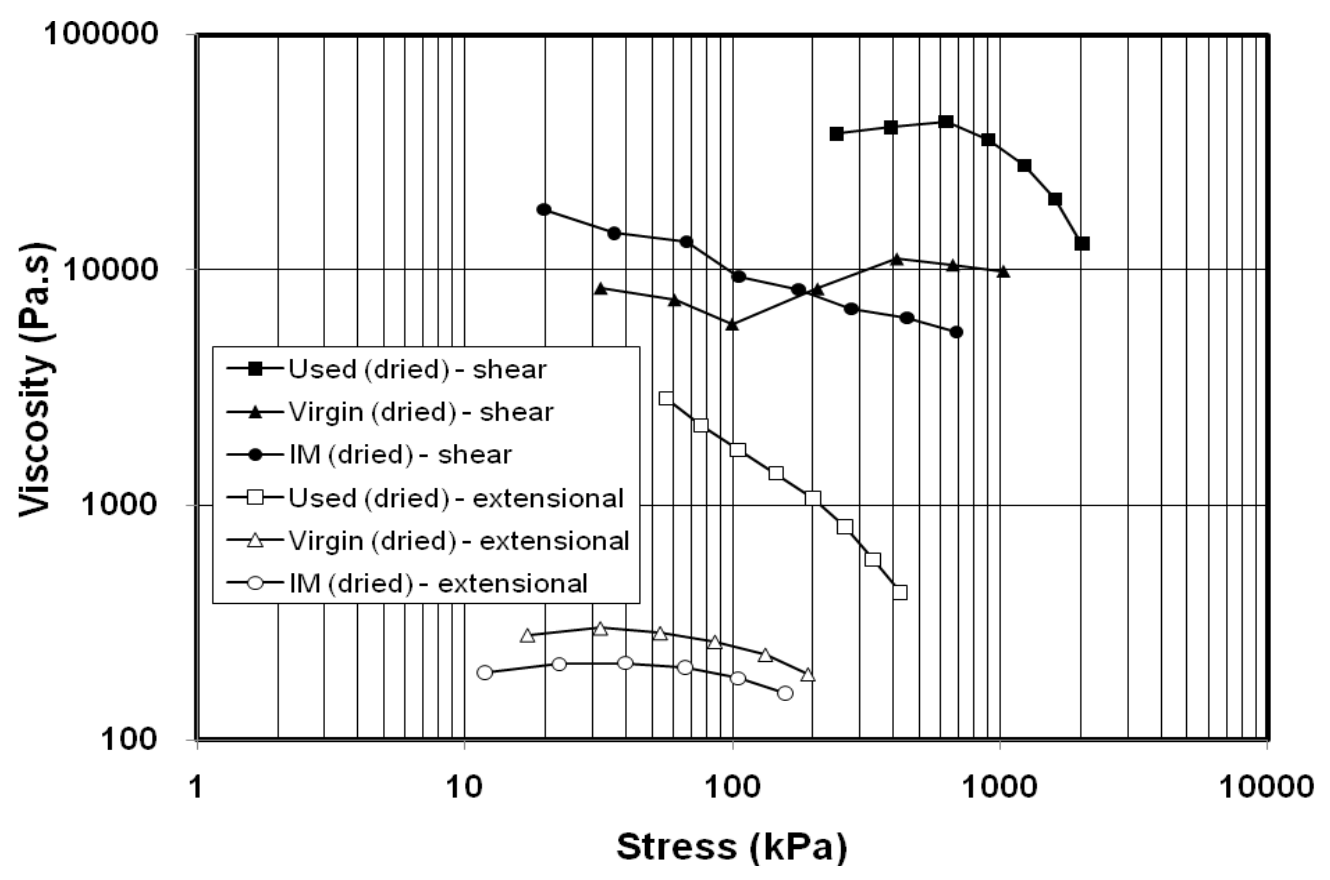

Figure 9 Composite plot of viscosity data for PA-12 polymers at $200{ }^{\circ} \mathrm{C}$ : shear and extensional flow data from capillary rheometry.

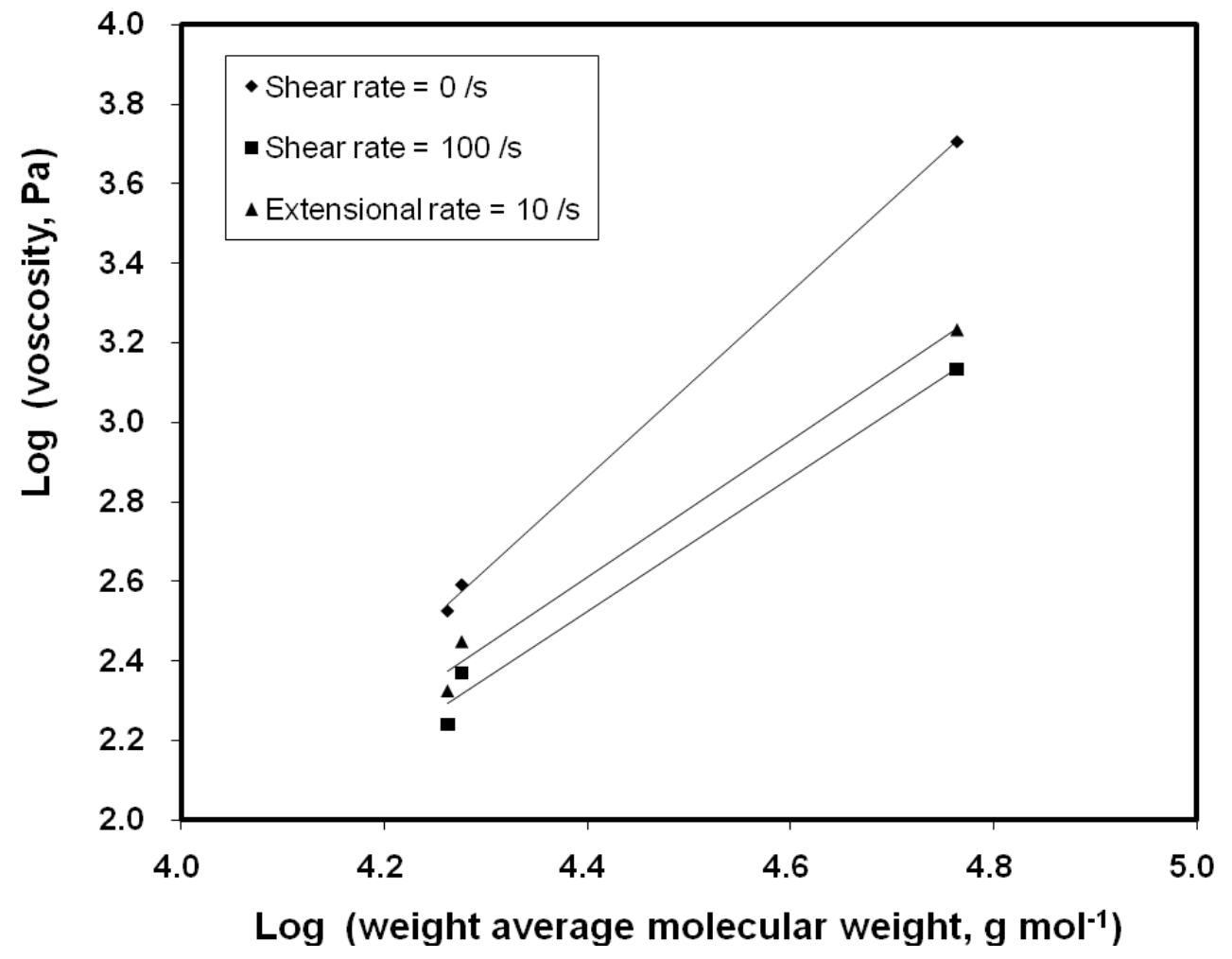

Figure 10 Zero shear viscosity versus Mw for PA-12 polymers. 


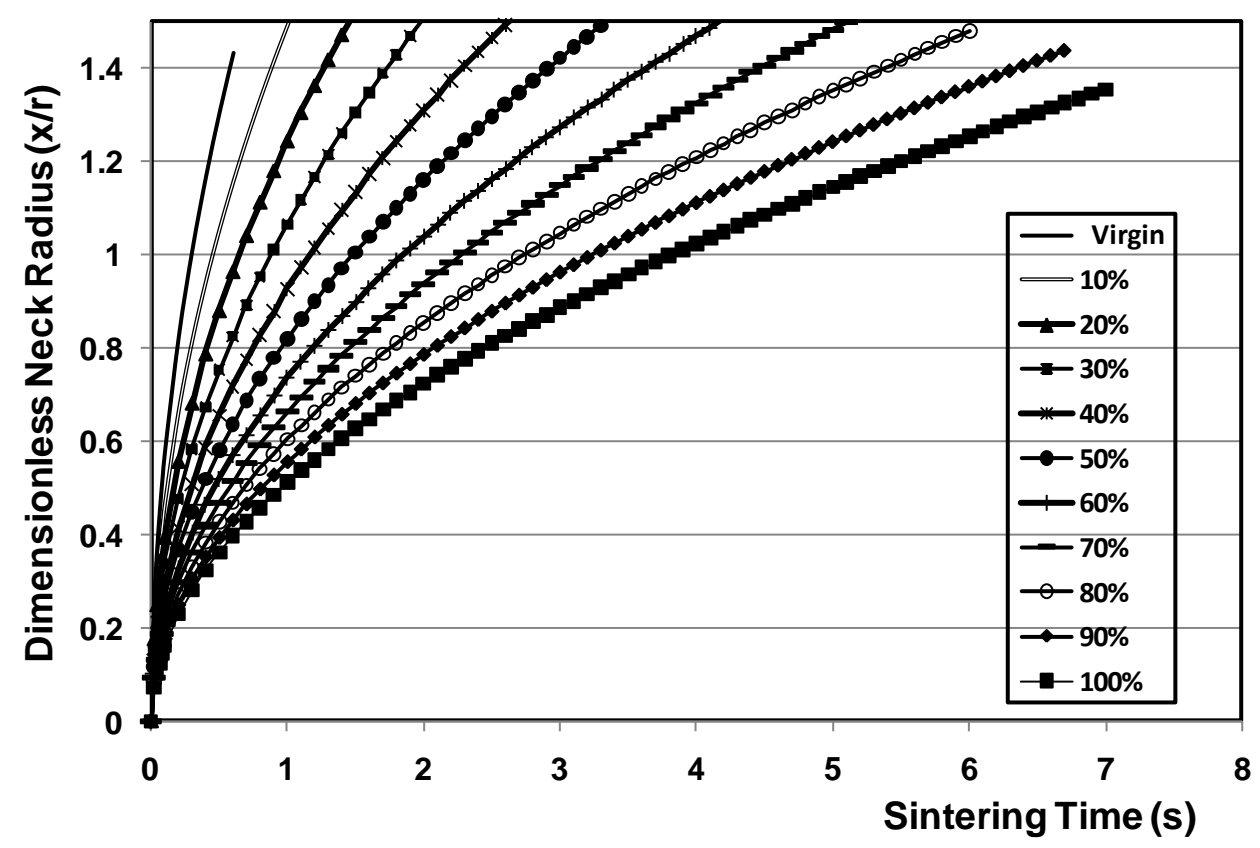

Figure 11 Frenkel model predictions, based upon viscosity / surface energy data: predicted sinter neck radius as a function of recycled PA12 (legend indicates the percentage of used PA-12 in the powder mix). 


\section{Tables}

Table I

PA-12 Materials used in Rheological Characterisation

\begin{tabular}{|c|c|c|c|c|}
\hline Name* & Grade / Trade name & Manufacturer & $\begin{array}{c}\text { Melting } \\
\text { point }\left({ }^{\circ} \mathbf{C}\right)\end{array}$ & $\begin{array}{c}\text { Physical } \\
\text { State }\end{array}$ \\
\hline PA 12 Virgin & PA 2200 & EOS GmbH & $172-180$ & Fine powder \\
\hline $\begin{array}{c}\text { PA 12 Virgin } \\
\text { (undried) }\end{array}$ & PA 2200 & EOS GmbH & $172-180$ & Fine powder \\
\hline $\begin{array}{c}\text { PA 12 } \\
\text { Used powder }\end{array}$ & N/A & EOS GmbH & N/A & Fine powder \\
\hline $\begin{array}{c}\text { PA 12 Injection } \\
\text { moulding (IM) }\end{array}$ & $\begin{array}{c}\text { AMNO } \\
\text { (Rilsan® Polyamide-12) }\end{array}$ & Arkema & $174-180$ & Granule \\
\hline
\end{tabular}

Table II Power law constants for PA12 polymers ( $k$ and $n$ ) obtained from capillary rheometry

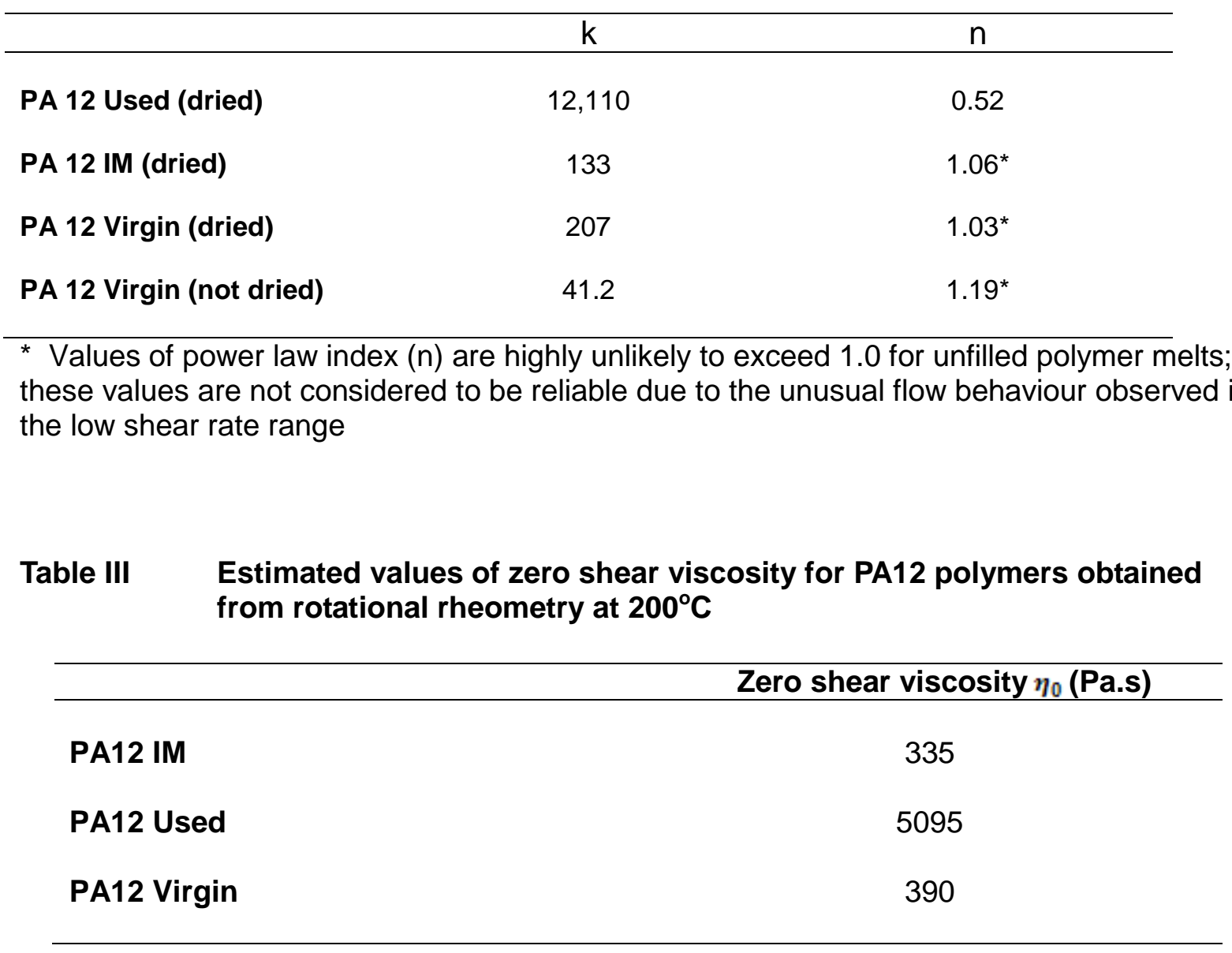


Table IV Molecular weight / distribution data for PA12 polymers obtained by GPC analysis

\begin{tabular}{lccc}
\hline & Mn $\left(\mathbf{g ~ m o l}^{-1}\right)$ & Mw $\left(\mathbf{g ~ m o l}^{-1}\right)$ & Mw/Mn \\
\hline PA12 IM & 6,090 & 18,300 & 3.0 \\
PA12 Used & 12,650 & 58,050 & 4.6 \\
PA12 Virgin & 6,175 & 18,900 & 3.1 \\
\hline
\end{tabular}

Table V Surface energy data from contact angle analysis at room temperature for PA-12 (virgin and used materials)

\begin{tabular}{lccc}
\hline & $\begin{array}{c}\text { Dispersive } \\
\text { component } \\
(\mathbf{m N} / \mathbf{m})\end{array}$ & $\begin{array}{c}\text { Polar component } \\
(\mathbf{m N} / \mathbf{m})\end{array}$ & $\begin{array}{c}\text { Surface energy } \\
(\mathbf{m N} / \mathbf{m})\end{array}$ \\
\hline PA-12 Virgin & 39.7 & 6.4 & 46.1 \\
PA-12 Used & 36.3 & 1.7 & 38.0 \\
PA-12 $\left(\mathbf{2 3}^{\circ} \mathbf{C}\right)^{*}$ & & & 31.0 \\
PA-12 $\left(\mathbf{m e l t}^{*}\right.$ & & 25.0 \\
PA-12 $\left(\mathbf{2 6 5}{ }^{\circ} \mathrm{C}\right)^{*}$ & & 22.0 \\
PA-12 ${ }^{*}$ & & $20.0-30.0$ \\
\hline
\end{tabular}

Surface energy is the sum of the dispersive and polar components.

Other data taken from literature: * Brandrup et al (1999); ** Shi et al (2004) 